\title{
GLOMANGIOMA: A RARE CASE REPORT
}

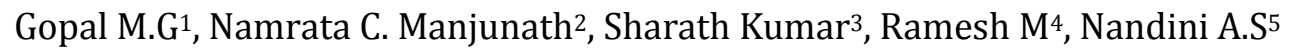

\section{HOW TO CITE THIS ARTICLE:}

Gopal M.G, Namrata C. Manjunath, Sharath Kumar, Ramesh M, Nandini A.S. “Glomangioma: A Rare Case Report”. Journal of Evolution of Medical and Dental Sciences 2014; Vol. 3, Issue 01, January 06; Page: 127-132.

ABSTRACT: Glomus tumors are benign localized tumors of the skin accounting for $1 \%$ to $2 \%$ of all soft tissue tumors. They may present as a solitary tumor or multiple tumors, termed glomangioma. They are usually solitary, small bluish, painful lesions usually located under the fingernails. They are more common in females than in males. Multiple lesions are slightly more common in males and they develop 10-15 years earlier than single lesions; about one third of the cases of multiple tumors occur in those younger than 20 years. Multiple tumors are less likely to be painful. Congenital glomus tumors are rare; they are plaque like in appearance and are considered a variant of multiple glomus tumors. Familial glomangiomas have been associated with a variety of deletions in the glomulin gene, and are inherited in an autosomal dominant manner, with incomplete penetrance. A 30 year-oldmale patient presented with asymptomatic multiple lesions of about 2 years duration. Clinical examination demonstrated numerous greenish blue nodules overchest, back, lower extremity. Histological examination revealed non-capsulated inflammatory infiltrate with multiple irregular vascular channels lined with endothelial cells. Multiple RBCs are also, present within the vascular channels consistent with glomangioma. We are reporting a rare case of glomangioma.

KEYWORDS: Glomangioma, glomus tumor.

INTRODUCTION: Glomangiomas are benign localized tumors of the skin characterized by abnormal, smooth muscle-like glomus cells. ${ }^{1}$ The term glomangioma was coined by Bailey ${ }^{2}$ in 1935 and is currently applied to lesions with a wide vascular lumen, which are most commonly found in patients with multiple tumors. ${ }^{3}$ Glomustumors arise from glomus cells, a thermoregulatory shunt concentrated in the fingers and toes, are uncommon, benign perivascular tumours. ${ }^{4}$ They have been categorized into subtypes, which include solitary, multiple, solid, diffuse, adult, and pediatric. ${ }^{5}$ More recently, these tumors have been classified into two major subtypes: solitary glomus tumors and multiple glomus tumors, which are also known as glomangiomas or glomuvenous malformations. ${ }^{6}$ Each variant has distinct clinical and histopathologic characteristics. Glomangiomas usually asymptomatic multiple pink-to-blue nodules or plaque like lesions. They occur in childhood and adolescence, and do not have a predilection for the subungal region.7-8 Multiple glomangiomas are rare and comprise about 10 percent of all glomus tumors. ${ }^{9}$

CASE REPORT:A 30 year-old male patient presented with multiple lesions of about 2 years duration. Lesions were occasionally associated with mild pain. The lesions initially presented on left lower extremity which gradually progressed to involve chest, back and loin on left side (Fig 1-2).He had no significant past history. He was non diabetic and non-hypertensive. No history of similar lesions in the family. No history of other systemic complaints.

General physical examination and systemic examination was normal. Clinical examination showed multiple greenish blue nodules of varying size from few $\mathrm{mm}$ to around $3 \mathrm{~cm}$ in diameter 


\section{CASE REPORT}

distributed adjacent to each other over an area on left thigh and loin. Numerous painless, few relatively larger ones were mildly tender on palpation present over back and lower extremity. Most of the nodules are firm in consistency, but some are partially compressible and non-tender to palpation or pressure. Mucous membranes not involved.

After history and clinical examination following differential diagnosis were made:

Glomangioma

Angioleiomyoma

Angiolipoma

Smooth muscle hamartoma

Neurofibroma

Kaposi sarcoma

An excision biopsy was taken from one of the nodules on the left thigh (Fig 3-4). Histological examination revealed non-capsulated inflammatory infiltrate with multiple irregular vascular channels lined with endothelial cells (Fig 5-6). The infiltrate composed mainly of monomorphic round or polygonal glomus cells with round or ovoid nucleus and scant eosinophilic cytoplasm. Several layers of these cells are present in the walls of vascular channels and in the adjacent stroma. Multiple RBCs are also, present within the vascular channels. (Fig 7).

With the clinical features and skin biopsy reports, final diagnosis of glomangioma was made. Patient was reassured and advised to come if there were any signs of malignancy.

DISCUSSION: Glomus tumors are thought to represent neoplastic proliferations of modified smooth muscle cells, called glomus cells, located in the walls of the Sucquet-Hoyer canal, a specialized arteriovenous anastomosis found most often in the fingers and play an important role in thermoregulation. ${ }^{10}$

Glomus tumors are classified into solid glomus tumors, glomangiomas, or glomangiomyomas according to the predominant histologic component present, that is, glomus cells, vascular spaces or blood vessels (angiomatoid), or smooth muscle fibers, respectively.

Solid Glomus tumors are usually present in young adults (ages 20-40) as a small, solitary, blue-red papule or nodule of the distal extremities, with a predilection for subungual sites. They are typically painful, often causing paroxysmal pain in response to temperature changes or pressure. ${ }^{10}$ Glomangiomas, also known as glomuvenous malformations, are a rare multiple variant accounting for $10 \%$ of cases, most often seen in children oradolescence, and in general show no gender predilection. ${ }^{11}$ Glomangiomas are thought to be inherited in an autosomal dominant pattern with incomplete penetrance and variable expression. Familial glomangiomas have been associated with a variety of deletions in the glomulin gene mapped to chromosome 1p21-p22. Glomangiomas are thought to be a result of loss of function mutations in the cytoplasmic protein glomulin. ${ }^{12}$

Glomangiomas are subdivided clinically into nodular and plaque-like lesions. They can vary in color from pink-to-blue and often become darker, and sometimes hperkeratotic with age. Interestingly, while glomus tumors predominate on the hands and fingers especially, glomangiomas can occur in a wide anatomic distribution, to include sites not known to contain glomus cells. Extracutaneous sites have been reported, including involvement of the gastrointestinal tract, 
trachea, nerve, bone, mediastinum, liver, pancreas, and ovary. One explanation for this finding is that these tumors may arise from pluripotent mesenchymal perivascular cells that can differentiate into glomus cells or even arise from ordinary smooth muscle cells. ${ }^{10}$

The prognosis for patients with glomangiomas is excellent, and most patients never experience any related medical problems.Malignant transformation (glomangiosarcomas) within glomus tumors is extremely rare and typically represents a locally infiltrative malignancy; however, metastases have been described and are associated with a very poor prognosis. ${ }^{13}$ Features of glomangiosarcomas may include the following. ${ }^{13}$

- Size larger than $2 \mathrm{~cm}$

- Rapid growth

- Deep soft tissue involvement

Glomangiomas differ clinically from glomus tumors in that they occur in childhood and adolescence, usually asymptomatic, do not have a predilection for the subungal region, and often are multifocal.7-8 Histologically, Glomangiomas are less well circumscribed (not capsulated) and less solid appearing than their solitary counterparts. They contain multiple irregular, dilated, endothelium- lined vascular channels that contain red blood cells. The vascular spaces are larger than those in a solitary glomus tumor. Small aggregates of cuboidal, glomus cells are present in the walls of these channels and in small clusters in the adjacent stroma. Each cell has a round or ovoid nucleus and scant eosinophilic cytoplasm. The overall appearance of multiple glomus tumors accounts for their alternate name; glomangiomas. ${ }^{5-8}$ Glomangiosarcomas resemble benign glomus tumors. However, glomangiosarcomas have more atypia, pleomorphism, and mitotic figures, and an invasive growth pattern. ${ }^{13}$ Tumor cells of glomangiomas are positive for $\alpha$-smooth muscle actin and vimentin but negative for CD31, CD34, von Wille brand factor, and S-100.6-7,12.Most cases are also negative for desmin, but rare desmin expression has been reported. ${ }^{14}$

Routine laboratory studies are not helpful in patients with glomangiomas. CBC count is required only in rare cases with widely disseminated lesions in which platelet sequestration is a concern. High-resolution is probably the criterion standard for the imaging of glomus tumors, followed by contrast-enhanced CT. Glomus tumors are strongly enhancing masses by both CT and MRI. ${ }^{15}$

Choosing the appropriate treatment regimen for glomus tumors and glomangiomas should be individualized to the patient and guided by the clinical presentation. Treatment is not always indicated, particularly in asymptomatic cases of glomangioma. Excision for multiple glomangiomasmay be difficult because of their poor circumscription, multifocal nature and the large number of lesions. So, excision should be limited to symptom aticlesions only. In a recent series of large facial glomangiomas, ${ }^{16}$ MRI was the best modality for definition of the extent of the lesions and their relationship to other anatomic structures. In this same series, surgical resection reduced the area of discoloration and improved facial contour. Other reported treatment modalities, more useful in treating multiple lesions, include argon and carbon dioxide laser therapy, electron-beam radiation and sclerotherapy with hypertonic saline or sodium tetradecyl sulfate. However, sclerotherapy was found to be less effective than for venous malformations. ${ }^{16}$ Periodic observation of asymptomatic lesions is usually required. ${ }^{5}$ 


\section{REFERENCES:}

1. Parsi K, Kossard S. Multiple hereditary glomangiomas: successful treatment with sclerotherapy. Aust J Dermatol. 2002; 43:43-47.

2. Bailey OT. The cutaneous glomus and its tumors-glomangiomas. Am J Pathol. 1935; 11:915936.

3. Monteagudo C, Carda C, Llombart-Bosch A, et al. Multiple glomangiomyoma versus glomangioma: conceptual and ultrastructural observations. Am J Dermato Pathol. 2000; 22:371-373.

4. Jon H Meyerle, MD, Dirk M Elston, MD, Erin L Spillane, MD, et al. Dermatologic Manifestations of Glomus Tumor, Medscape Dermatology, 2010.

5. Marie Leger MD PhD, Utpal Patel MD PhD, Rajni Mandal MD, et al. Glomangioma, Dermatology Online Journal 2010; 16 (11):11.

6. Bolognia J, et al. eds. Dermatology, 2nd edition, London: Mosby, 2008: 114:1790.

7. McEvoy BF, et al. Multiple hamartomatous glomus tumors of the skin. Arch Dermatol 1971; 104:188.

8. Moor EV, et al. Multiple glomus tumors: a case report and review of the literature. Ann Plast Surg 1999; 43:436.

9. Goodman TE, Abele DC. Multiple glomus tumors. Arch Dermatol 1971; 103:11.

10. Jon H Meyerle, MD, Dirk M Elston, MD, Erin L Spillane, MD, et al. Dermatologic Manifestations of Glomus Tumor, Medscape Dermatology, 2010.

11. Mounayer C, Wassef M, Enjolras O, et al. Facial "glomangiomas": large facial venous malformations with glomus cells. J Am Acad Dermatol. 2001; 45:239-45.

12. Brouillard $\mathrm{P}$, et al. Mutations in a novel factor, glomulin is responsible for glomuvenous malformations ("glomangiomas"). Am J Hum Genet 2002; 70:866?

13. Folpe AL, Fanburg-Smith JC, Miettinen M, Weiss SW. Atypical and malignant glomus tumors: analysis of 52 cases, with a proposal for the reclassification of glomus tumors. Am J Surg Pathol. Jan 2001; 25(1):1-12. [Medline].

14. Porter PL, Bigler SA, McNutt M, et al. The immunophenotype of hemangiopericytomas and glomus tumours, with special reference to muscle protein expression: an immunohistochemical study and review of the literature. Mod Pathol. 1991; 4:46-52

15. Drape JL, Idy-Peretti I, Goettmann S, Guerin-Surville H, Bittoun J. Standard and high resolution magnetic resonance imaging of glomus tumors of toes and fingertips. J Am AcadDermatol. Oct 1996; 35(4):550-5.

16. Enjolras O, Ciabrini D, Mazoyer E, Laurian C, Herbreteau D. Extensive pure venous malformations in the upper or lower limb: a review of 27 cases. J Am Acad Dermatol. 1997; $36: 219-25$. 


\section{CASE REPORT}

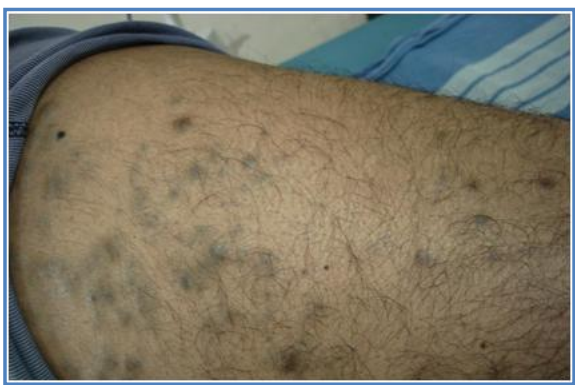

Fig. 1: Multiple glomus nodules on the left thigh

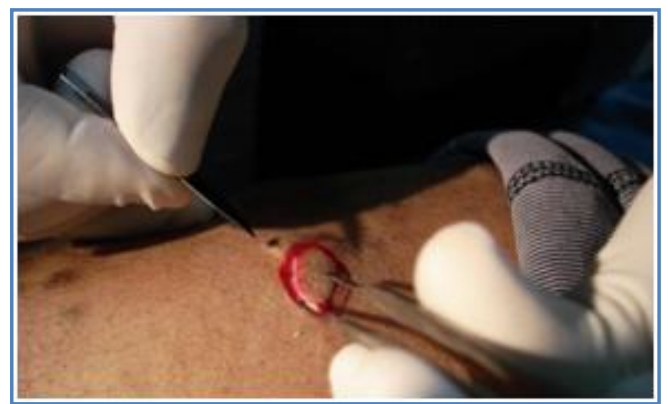

Fig. 3 and 4: Excision biopsy from nodule on left thigh
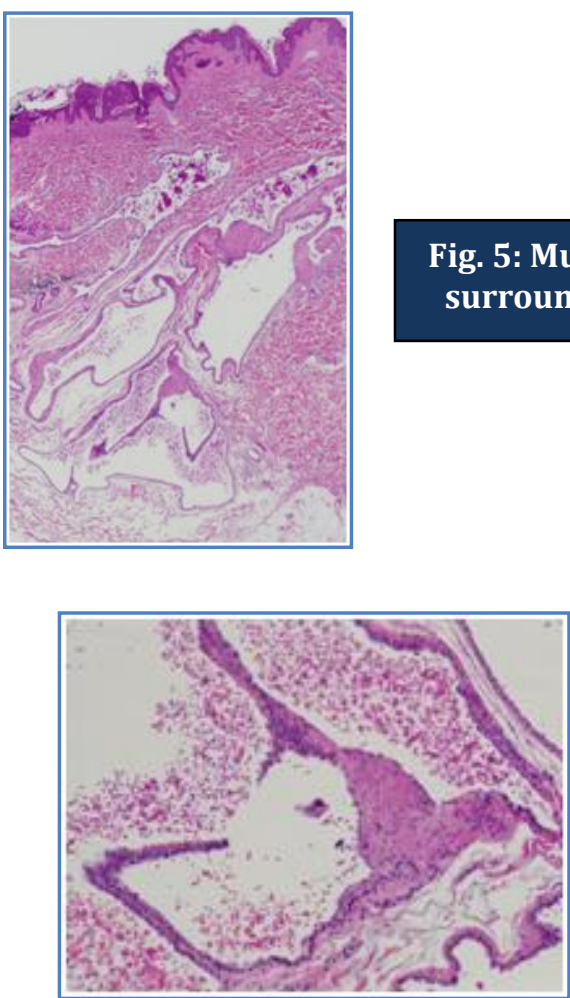

Fig. 6: Vascular channels are lined with endothelial cells that are coated with glomus cells.

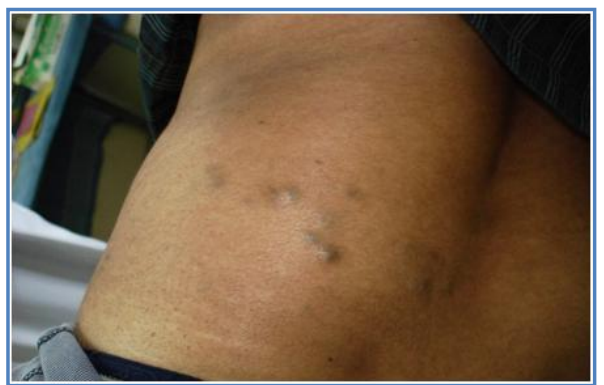

Fig. 2: Multiple glomus nodules on the left loin.

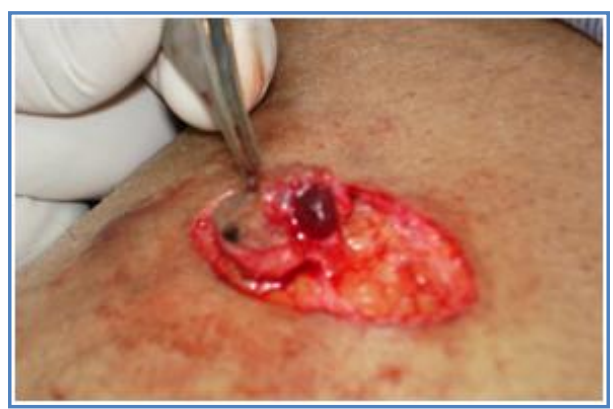

Fig. 5: Multiple, irregular vascular channels surrounded with inflammatory infiltrate. 


\section{AUTHORS:}

1. Gopal M.G.

2. Namrata C. Manjunath

3. Sharath Kumar

4. Ramesh M

5. Nandini A.S.

\section{PARTICULARS OF CONTRIBUTORS:}

1. Professor and HOD, Department of Dermatology and STD, Kempegowda Institute of Medical Sciences.

2. Post Graduate Student, Department of Dermatology and STD, Kempegowda Institute of Medical Sciences.

3. Professor, Department of Dermatology and STD, Kempegowda Institute of Medical Sciences.
4. Associate Professor, Department of Dermatology and STD, Kempegowda Institute of Medical Sciences.

5. Assistant Professor, Department of Dermatology and STD, Kempegowda Institute of Medical Sciences.

\section{NAME ADDRESS EMAIL ID OF THE} CORRESPONDING AUTHOR:

Dr.Namrata C. Manjunath, \# 281, 80 Feet Road, Padmanabhanagar, Bangalore - 560070 .

Email-drnamratamanjunath@gmail.com

Date of Submission: 16/12/2013.

Date of Peer Review: 17/12/2013.

Date of Acceptance: 26/12/2013.

Date of Publishing: 02/01/2014 